\title{
Ontology-based knowledge model for virtual communities profile
}

\begin{abstract}
Knowledge management (KM) system is develop to support the organizational knowledge process of knowledge acquisition, knowledge organization, knowledge dissemination and knowledge application. The KM system must be able to support virtual communities (VC) of an organization. In order to determine the potential member of $\mathrm{VC}$, the $\mathrm{KM}$ system should define, identify and structure $\mathrm{VC}$ based on its profile and knowledge. The process of $\mathrm{VC}$ classification based on knowledge and profile are not an easy process. The ontology based knowledge model will become essential in classifying and structuring knowledge of VC in an organization and many organizations has recognized that knowledge is a powerful asset. The aim of the paper is to analyze and promote the ontology based knowledge model VC profile in $\mathrm{KM}$ system so that the process of $\mathrm{VC}$ classification can be done. And later, the ontology based knowledge model VC profile can be use to standardize the classification of VC in KM system. The model can be reused and shared for other purposes. The knowledge models were developed and applied in the research and development (R\&D) as a case study. Finally, the study will provide a holistic view that combines both usability and sociability factors in which often isolated when developing a KM system.
\end{abstract}

Keyword: Virtual communities profile; Knowledge model; Ontology; KM system; Ontology based VC model 